NISSUNA UMANA INVESTIGAZIONE SI PUO DIMANDARE VERA SCIENZIA S'ESSA NON PASSA PER LE MATEMATICHE DIMOSTRAZIONI LEONARDO DA VINCI

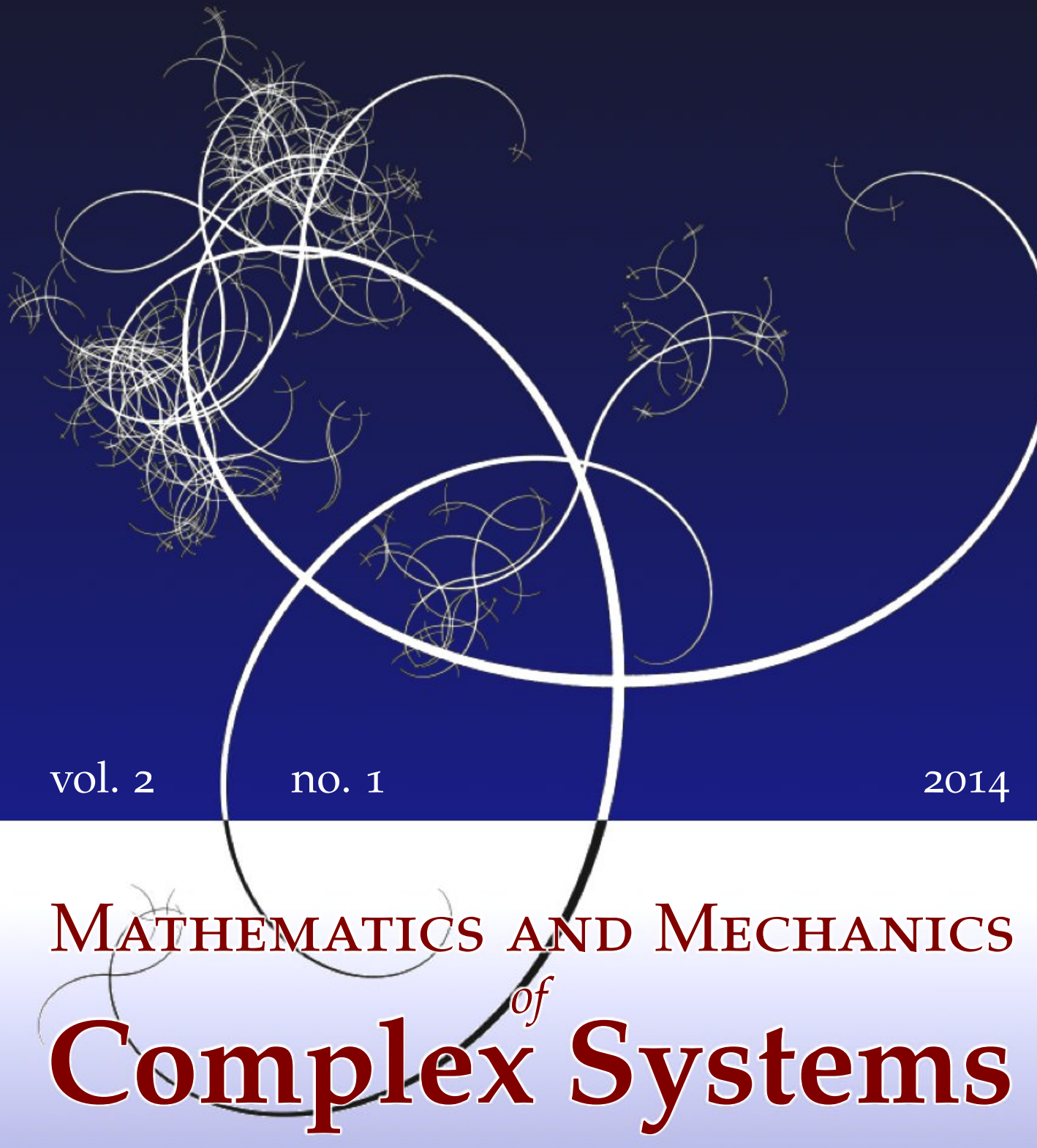

HENRI GOUIN

INTERFACES ENDOWED WITH

NONCONSTANT SURFACE ENERGIES REVISITED WITH THE D'ALEMBERT-LAGRANGE PRINCIPLE 


\title{
INTERFACES ENDOWED WITH NONCONSTANT SURFACE ENERGIES REVISITED WITH THE D'ALEMBERT-LAGRANGE PRINCIPLE
}

\author{
HENRI GOUIN
}

\begin{abstract}
The equation of motion and the conditions on surfaces and edges between fluids and solids in the presence of nonconstant surface energies, as in the case of surfactants attached to fluid particles at the interfaces, are revisited under the principle of virtual work. We point out that adequate behaviors of surface concentrations may drastically modify the surface tension which naturally appears in the Laplace and the Young-Dupré equations. Thus, the principle of virtual work points out a strong difference between the two revisited concepts of surface energy and surface tension.
\end{abstract}

\section{Introduction}

This paper develops the principle of virtual work due to d'Alembert-Lagrange [Serrin 1959 $]^{1}$ when different phases of fluids are in contact through singular surfaces or interfaces. The study is first presented without a constitutive assumption for the surface energies, but the displacement fields are considered for a simple material corresponding to the first-gradient theory. The d'Alembert-Lagrange principle allows us to obtain the equation of motion and boundary conditions of mechanical nature and is able to be extended to more complex materials with microstructures [Daher and Maugin 1986] or to multigradient theories [Gouin 2007]. Here, we aim to emphasize the formulation of the principle of virtual work when the interfaces are endowed with nonconstant surface energies: the surfaces have their own material properties independent of the bulks and are embedded in the physical space, which is a three-dimensional metric space. The surface energy density is taken into account and naturally comes into the boundary conditions as the Laplace and the Young-Dupré equations by using variations associated with the virtual displacement fields. To do so, it is necessary to propose a constitutive equation of the surface energy; defining this is a main purpose of the paper. Such a

PACS2010: $02.30 . \mathrm{Xx}, 45.20 . \mathrm{dg}, 68.03 . \mathrm{Cd}, 68.35 . \mathrm{Gy}$.

Keywords: variational methods, capillarity, surface energy, surface tension.

${ }^{1}$ The principle of virtual work is also referred to in the literature as the principle of virtual power while virtual displacements are called virtual velocities [Germain 1973a; 1973b]. 
presentation is similar that of deformational and configurational mechanics [Steinmann 2008]; the method is analogous with the one employed in [Germain 1973a; 1973b; Daher and Maugin 1986] but with powerful differential geometry tools as in [Fosdick and Tang 2009]. However, the mathematical tools are adapted to the linear functional of virtual displacement fields and not to the integral balance laws over nonmaterial interfaces separating fluid phases as in [Cermelli et al. 2005].

Consequently, the main result of this paper is to propose a general form of the linear functional with interfaces in first-gradient theory which points out the significance of constitutive behaviors for the surface energies and highlights the clear difference between the notions of surface energy and surface tension. Fischer et al. [2008] emphasized a thermodynamical definition of surface energy, surface tension, and surface stress for which surface tension and surface stress are identical for fluids. Our presentation is not the same: without any thermodynamical assumptions, the difference between surface energy and surface tension is a natural consequence of the virtual work functional and the d'Alembert-Lagrange principle. The surface energy allows us to obtain the total energy of the interfaces and the surface tension is directly generated from the boundary conditions of the continuous medium.

In the simplest cases the two notions of surface energy and surface tension are mingled, but this is not generally the case when the surface energy is nonconstant along the interfaces. To prove this property, we first focus on the simplest case of Laplace's capillarity and obtain the well-known equations on interfaces and contact lines.

Surfaces endowed with surface matter, as in the case of surfactants, are a more complex case. Recent decades have seen the extension of surfactant applications in many fields including biology and medicine [Rosen 2004]; surfactants can also be expected to play a major mechanical role in the fluid and solid domains. The versatility of a surfactant mainly depends on its concentration at interfaces. It experimentally appears that a surfactant or surface-active agent is a substance present in liquids at a very low concentration rate and, when surface mass concentration is below the critical micelle concentration, it is mainly absorbed onto interfaces and alters only the interfacial free energies [de Gennes et al. 2004]. The interfacial free energy per unit area (generally called the surface energy) is the minimum amount of work required to create an interface at a given temperature [Edwards et al. 1991; Slattery et al. 2007]. The fact that surfactants can affect the mechanical behaviors of interfaces must be modelized in order to predict and control the properties of complete systems.

In fact, our aim is not to study the general case of surfactants proposed in the literature but to focus on the virtual work method to prove that simple behaviors of the surface energy depending on the mass concentration can drastically change the capillary effects. So, the concept of surface tension naturally appears in the 
equations on surfaces and on lines. In this paper, we call a surfactant the matter distributed only on the interfaces: we consider the special case when surfactant molecules are insoluble in the liquid bulk (the surface mass concentration is below the critical micelle concentration [Rosen 2004]) and are attached to fluid particles along the interfaces (without surface diffusion as in [McBride et al. 2011]).

The manuscript is organized as follows: Section 2 briefly reminds us of some results formally presenting the principle of virtual work in its more general form by using the kinematics of a continuous medium and the notion of virtual displacement. The simplest example of the Laplace model of capillarity concludes the section.

Section 3 deals with the case when the interfaces are endowed with nonconstant surface energy, whereby we essentially focus on liquid in contact with solid and gas. The special case of surfactants as interface matter attached to the fluid particles is considered. The surface energy depends on the surface matter concentration. Such a property drastically changes the boundary conditions on the interface by using surface tension instead of surface energy.

Section 4 deals with an explicit comparison between surface energy and surface tension only within deformational mechanics. And Section 5 is the conclusion in which some general extension can be forecast.

The main mathematics tools are collected in the large Appendix so that the presentation of the text is not cluttered with tedious calculations. The main mathematical tool is (15), which can be extended to more complex media.

\section{Virtual work for a continuous medium}

In continuum mechanics, motions can be equivalently studied with either the Newtonian model of a system offorces or the Lagrangian model of the work offorces [Germain 1973a; 1973b]. The Lagrangian model does not derive from a variational approach but, at equilibrium, the minimization of the energy coincides with the zero value of a linear functional. Generally, the linear functional expressing the work of forces is related to the theory of distributions; a decomposition theorem associated with displacements (as $C^{\infty}$-test functions whose supports are compact manifolds) uniquely determines a canonical zero order form (a separated form) with respect to both the test functions and the transverse derivatives of the contact test functions [Schwartz 1966]. In the same way that the Newtonian principle is useless when we do not have any constitutive equation for the system of forces, the d'AlembertLagrange principle is useless when we do not have any constitutive assumption for the virtual work functional.

The equation of motion and boundary conditions of a continuous medium derive from the d'Alembert-Lagrange principle of virtual work, which is an extension of the same principle in the mechanics of systems with a finite number of degrees of 
freedom: For any virtual displacement, the motion is such that the virtual work of forces is equal to the virtual work of mass accelerations [Gouin 2007].

2.1. The background of the principle of virtual work. The motion of a continuous medium is classically represented by a continuous transformation $\varphi$ of a threedimensional space into the physical set. In order to describe the transformation analytically, the variables $\boldsymbol{X}=\left(X^{1}, X^{2}, X^{3}\right)$ which single out individual particles correspond to material or Lagrange variables; the variables $\boldsymbol{x}=\left(x^{1}, x^{2}, x^{3}\right)$ correspond to Euler variables. The transformation representing the motion of a continuous medium is of the form

$$
\boldsymbol{x}=\boldsymbol{\varphi}(\boldsymbol{X}, t) \quad \text { or } \quad x^{i}=\varphi^{i}\left(X^{1}, X^{2}, X^{3}, t\right), \quad i \in\{1,2,3\},
$$

where $t$ denotes the time. At a fixed time the transformation possesses an inverse and continuous derivatives up to the second order except on singular surfaces, curves, or points. Then, the diffeomorphism $\varphi$ from the set $D_{0}$ of the particle references into the physical set $D$ is an element of a functional space $\wp$ of the positions of the continuous medium considered as a manifold with an infinite number of dimensions.

To formulate the d'Alembert-Lagrange principle of virtual work in continuum mechanics, we recall the notion of virtual displacements. This notion is obtained by letting the displacements arise from variations in the paths of particles. Let a one-parameter family of varied paths or virtual motions denoted by $\left\{\boldsymbol{\varphi}_{\eta}\right\}$, and possessing continuous partial derivatives up to the second order, be analytically expressed by the transformation

$$
\boldsymbol{x}=\boldsymbol{\Phi}(\boldsymbol{X}, t ; \eta),
$$

with $\eta \in O$, where $O$ is an open real set containing 0 , and such that $\boldsymbol{\Phi}(\boldsymbol{X}, t ; 0)=$ $\varphi(X, t)$ (the real motion of the continuous medium is obtained when $\eta=0$ ). The derivative with respect to $\eta$ at $\eta=0$ is denoted by $\delta$. In the literature, the derivative $\delta$ is named the variation and the virtual displacement is the variation of the position of the medium [Serrin 1959]. The virtual displacement is a tangent vector to $\wp$, the functional space of positions, at $\varphi\left(\delta \varphi \in T_{\varphi}(\wp)\right)$. In the physical space, the virtual displacement $\delta \varphi$ is determined by the variation of each particle: the virtual displacement $\zeta$ of the particle $\boldsymbol{x}$ is such that $\zeta=\delta \boldsymbol{x}$ when at $\eta=0,\{\delta \boldsymbol{X}=0, \delta t=0$, $\delta \eta=1\}$, and we associate the field of tangent vectors to $D$ :

$$
x \in D \quad \rightarrow \quad \zeta=\left.\psi(x) \equiv \frac{\partial \Phi}{\partial \eta}\right|_{\eta=0} \in T_{x}(D),
$$

where $T_{\boldsymbol{x}}(D)$ is the tangent vector bundle to $D$ at $\boldsymbol{x}$.

The virtual work concept, the dual of Newton's method, can be written in the following form: 

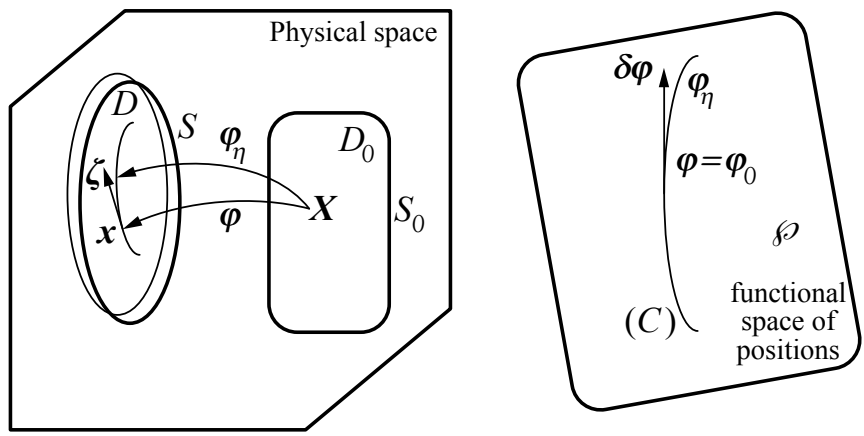

Figure 1. The boundary $S$ of $D$ is represented by a thick curve and its variation by a thin curve. The variation $\delta \varphi$ of the family $\left\{\varphi_{\eta}\right\}$ of varied paths belongs to $T_{\varphi}(\wp)$, tangent space of $\wp$ at $\varphi$.

The virtual work $\delta \tau$ is a linear functional value of the virtual displacement,

$$
\delta \tau=\langle\Im, \delta \varphi\rangle,
$$

where $\langle\cdot, \cdot\rangle$ denotes the inner product of $\Im$ and $\delta \varphi$, with $\Im$ belonging to the cotangent space $T_{\varphi}^{*}(\wp)$ of $\wp$ at $\varphi$.

In (3), the medium in position $\varphi$ is submitted to covector $\Im$ denoting all the "stresses" in mechanics. In the case of motion, we must add the inertial forces, corresponding to the accelerations of masses, to the volume forces.

The d'Alembert-Lagrange principle of virtual work is expressed as follows:

For all virtual displacements, the virtual work is null.

This principle leads to the analytic representation

$$
\forall \delta \varphi \in T_{\varphi}(\wp), \quad \delta \tau=0 .
$$

Theorem. If (3) is a distribution expressed in separated form (see [Schwartz 1966]), the d'Alembert-Lagrange principle yields the equation of motion and boundary conditions in the form $\mathfrak{\Im}=0$.

The virtual displacement is submitted to constraints coming from the constitutive equations and geometrical assumptions such as mass conservation. Consequently, the constraints are not expressed by Lagrange multipliers but are directly taken into account by the variations of the constitutive equations. The equation of motion and boundary conditions result from the explicit expression of $\delta \tau$ associated with the considered physical problem. As a first example, the simplest case of the theory of capillarity at equilibrium is considered. 
2.2. The classical Laplace theory of capillarity. Liquid-vapor and two-phase interfaces are represented by material surfaces endowed with an energy related to the Laplace free energy of capillarity. When working far from critical conditions, the capillary layer has a thickness equivalent to a few molecular beams [Ono and Kondo 1960; Domb 1996] and the interface appears as a geometrical surface separating the two media, with its own characteristic behavior and energy properties [Levitch 1962]. The domain $D$ of a compressible fluid (liquid) is immersed in a three Euclidean space. The boundary of the domain $D$ is a surface $S$ shared in $N$ parts $S_{p}$ of class $C^{2}, p=1, \ldots, N$ (Figure 2). We denote by $\left(R_{m}\right)^{-1}$ the mean curvature of $S$; the union of the limit edges $\Gamma_{p q}$ between surfaces $S_{p}$ and $S_{q}$ is assumed to be of class $C^{2}$ and $\boldsymbol{t}_{p}$ is the tangent vector to $\Gamma_{p}=\bigcup \Gamma_{p q}, q=1, \ldots, N$ with $q \neq p$, oriented by the unit external vector to $D$ denoted $\boldsymbol{n}_{p} ; \boldsymbol{n}_{p}^{\prime}=\boldsymbol{t}_{p} \times \boldsymbol{n}_{p}$ is the unit external normal vector to $\Gamma_{p}$ in the tangent plane to $S_{p}$; the edge $\Gamma$ of $S$ is the union of the edges $\Gamma_{p}$ of $S_{p}$.

To first verify the well-foundedness of the model, we consider the explicit expression of the functional $\delta \tau$ for compressible fluids with capillarity in the nondissipative case. The variation of the total energy $E$ of such a fluid results from the variation of the sum of the local density of energy integrated on the domain $D$ and the variation of the local density of surface energy integrated on its boundary $S$; to these variations, we must add the work of volume force $\rho \boldsymbol{f}$ in $D$, surface force $\boldsymbol{T}$ on $S$, and line force $\boldsymbol{L}$ on $\Gamma$. Such an amount represents, for the domain $D$, the virtual work of forces of the compressible fluid with capillarity.

The Laplace theory of capillarity introduces the notion of surface energy (or superficial energy) on surfaces such that, for a compressible liquid with capillary effects on the wall boundaries, the total energy of the fluid writes in the form

$$
E=\iiint_{D} \rho \alpha(\rho) d v+\iint_{S} \sigma d s, \quad \text { with } \iint_{S} \sigma d s \equiv \sum_{p=1}^{N} \iint_{S_{p}} \sigma_{p} d s
$$
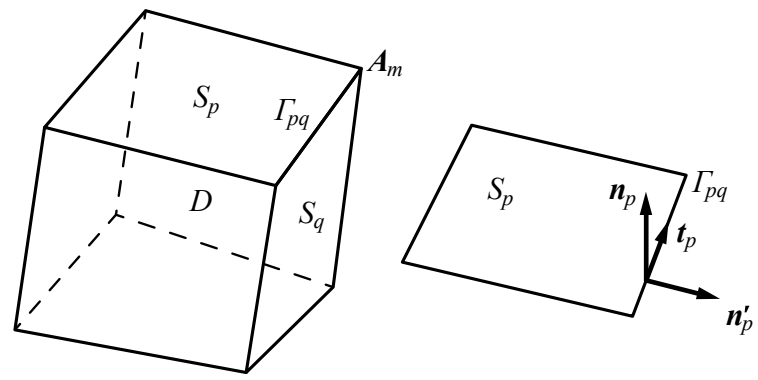

Figure 2. The set $D$ has a surface boundary $S$ divided into several parts. The edge of $S$ is denoted by $\Gamma$ which is also divided into several parts with endpoints $\boldsymbol{A}_{m}$. 
where $\rho$ is the matter density, $\alpha(\rho)$ is the fluid-specific energy ( $\rho \alpha(\rho)$ is the volume energy), and the coefficients $\sigma_{p}$ are the surface energy densities on each surface $S_{p}$ represented - for the sake of simplicity — by $\sigma$ on $S .^{2}$ Surface integrations are associated with the metric space. As proved in the Appendix, the variation of the deformation gradient tensor $F=\partial \boldsymbol{x} / \partial \boldsymbol{X}$ (with components $\left\{\partial x_{i} / \partial X_{j}\right\}$ ) of the mapping $\varphi$ combined with the mass conservation and the variation of $\sigma$ allow us to obtain the variation $-\delta E$ (see (A.3)); then the independent variables come from the position $\boldsymbol{x}$ of the continuous medium.

The virtual work of the volume forces defined on $D$ is generally in the form

$$
\iiint_{D} \rho \boldsymbol{f}^{T} \zeta d v, \quad \text { with } \boldsymbol{f}=-\operatorname{grad} U
$$

where $U(\boldsymbol{x})$ is a potential per unit mass and superscript $T$ denotes transposition. The virtual work of surface and line forces defined on $S$ and $\Gamma$ are, respectively,

$$
\iint_{S} \boldsymbol{T}^{T} \zeta d s \text { and } \quad \int_{\Gamma} \boldsymbol{L}^{T} \zeta d l
$$

Consequently, the total virtual work of forces $\delta \tau$ is

$$
\delta \tau=-\delta E+\iiint_{D} \rho \boldsymbol{f}^{T} \zeta d v+\iint_{S} \boldsymbol{T}^{T} \zeta d s+\int_{\Gamma} \boldsymbol{L}^{T} \zeta d l .
$$

From (A.3) and (A.6), we obtain

$$
\begin{aligned}
\delta \tau \equiv \iiint_{D}( & \left.-\operatorname{grad}^{T} p+\rho \boldsymbol{f}^{T}\right) \zeta d v+\int_{\Gamma}\left(\boldsymbol{L}^{T}-\sigma \boldsymbol{n}^{\prime T}\right) \zeta d l \\
& +\iint_{S}\left[-\delta \sigma+\left\{\left(p+\frac{2 \sigma}{R_{m}}\right) \boldsymbol{n}^{T}+\operatorname{grad}^{T} \sigma\left(\mathbf{1}-\boldsymbol{n n} \boldsymbol{n}^{T}\right)+\boldsymbol{T}^{T}\right\} \zeta\right] d s,
\end{aligned}
$$

where $p \equiv \rho^{2} \alpha^{\prime}(\rho)$ is the pressure of the liquid [Rocard 1952], $\delta \sigma$ denotes the variation of the surface energy $\sigma$, and $\mathbf{1}$ denotes the identity tensor. When $\sigma$ is constant we get $\delta \sigma=0$; then,

$$
\begin{aligned}
\delta \tau \equiv \iiint_{D}\left(-\operatorname{grad}^{T} p+\right. & \left.\rho \boldsymbol{f}^{T}\right) \zeta d v \\
& +\iint_{S}\left\{\left(p+\frac{2 \sigma}{R_{m}}\right) \boldsymbol{n}^{T}+\boldsymbol{T}^{T}\right\} \zeta d s+\int_{\Gamma}\left(\boldsymbol{L}^{T}-\sigma \boldsymbol{n}^{\prime T}\right) \zeta d l,
\end{aligned}
$$

and the d'Alembert-Lagrange principle yields the equation of equilibrium on $D$,

$$
-p_{, i}+\rho f_{i}=0 \quad \text { or } \quad-\operatorname{grad} p+\rho \boldsymbol{f}=0 .
$$

${ }^{2}$ Our aim is not to consider the thermodynamics of interfaces. Consequently, $\alpha$ and $\sigma$ are not considered as functions of thermodynamical variables such as temperature or entropy. 
The condition on boundary surface $S$ is

$$
\left(p+\frac{2 \sigma}{R_{m}}\right) n_{i}+T_{i}=0 \quad \text { or } \quad\left(p+\frac{2 \sigma}{R_{m}}\right) \boldsymbol{n}+\boldsymbol{T}=0,
$$

where, for an external fluid bordering $D, \boldsymbol{T}=-P \boldsymbol{n}$, with $P$ the value of the pressure in the external fluid. On the lines, it is necessary to consider the partition of $S$ such that the edge $\Gamma_{p q}$ is common to $S_{p}$ and $S_{q}$,

$$
\sigma_{p} n_{p i}^{\prime}+\sigma_{q} n_{q i}^{\prime}-L_{i}=0 \quad \text { or } \quad \sigma_{p} \boldsymbol{n}_{p}^{\prime}+\sigma_{q} \boldsymbol{n}_{q}^{\prime}-\boldsymbol{L}=0 .
$$

Surface condition (6) is the Laplace equation and line condition (7) is the YoungDupré equation with a line tension $\boldsymbol{L}$.

It is interesting to note that Steigmann and $\mathrm{Li}$ [1995] used the principle of virtual work by utilizing a system of line coordinates on boundary surfaces and lines. By introducing the free energy per unit area of interfaces and the free energy per unit of contact curve, they obtained Laplace's equation and a generalization of the YoungDupré equation of equilibrium; moreover, by employing the necessary conditions for energy-minimizing states of fluid systems they got a demonstration that the line tension associated with a three-phase contact curve must be nonnegative.

When $\sigma$ is not constant but $\delta \sigma=0$, we obtain the same equations for (5) and (7) but (6) on $S$ is replaced by

$$
\left(p+\frac{2 \sigma}{R_{m}}\right) \boldsymbol{n}+\left(\mathbf{1}-\boldsymbol{n} \boldsymbol{n}^{T}\right) \operatorname{grad} \sigma+\boldsymbol{T}=0 .
$$

The additive term $\left(\mathbf{1}-\boldsymbol{n} \boldsymbol{n}^{T}\right) \operatorname{grad} \sigma=\operatorname{grad}_{t g} \sigma$ is the tangential part of $\operatorname{grad} \sigma$ to the surface $S$. This term corresponds to a shear stress necessarily balanced by the tangential component of $\boldsymbol{T}$. Such is the case when $\sigma$ is defined on the $S_{0}$ image of $S$ in the reference space $D_{0}$ (then, $\sigma=\sigma_{0}(\boldsymbol{X})$ ). We understand the importance of the surface energy constitutive behavior; this questioning is emphasized in the following section.

\section{Capillarity of liquid in contact with solid and gas in the presence of nonconstant surface energy}

We have seen in the previous section that the problem associated with the behavior of the surface energy is the key point to obtaining the boundary conditions on interfaces and contact lines bordering the fluid bulk. In this section we consider a very special case of surfactant: the interfaces are endowed with a concentration of matter which affects the surface energy. The surface matter is attached to the particles of the fluid such that they obey together to the same equations of motion (1) and of virtual motion (2). We consider a more general case than in Section 2.2: 


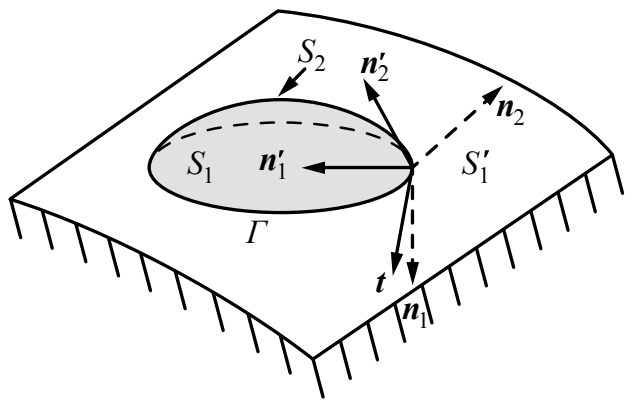

Figure 3. A liquid in drop form lies on a solid surface. The liquid is bordered by a gas and a solid; $S_{1}$ is the boundary between liquid and solid; $S_{1}^{\prime}$ is the boundary between gas and solid; $S_{2}$ is the interface between liquid and gas; $\boldsymbol{n}_{1}$ and $\boldsymbol{n}_{2}$ are the unit normal vectors to $S_{1}$ and $S_{2}$, external to the domain of the liquid; the edge $\Gamma$ (or contact line) is common to $S_{1}$ and $S_{2}$ and $t$ is the unit tangent vector to $\Gamma$ relative to $\boldsymbol{n}_{1} ; \boldsymbol{n}_{1}^{\prime}=\boldsymbol{n}_{1} \times \boldsymbol{t}$ and $\boldsymbol{n}_{2}^{\prime}=\boldsymbol{n}_{2} \times(-\boldsymbol{t})$ are the binormals to $\Gamma$ relative to $S_{1}$ and $S_{2}$, respectively.

we study the motion of the continuous medium with viscous forces. This viscosity affects not only the equation of motion but also the boundary conditions.

3.1. Geometrical description of the continuous medium. A drop of liquid fills the set $D$ and lies on the surface of a solid. The liquid drop is also bordered by a gas. All the interfaces between liquid, solid and gas are assumed to be regular surfaces. We call $\sigma_{S_{1}}$ and $\sigma_{S_{2}}$ the values of the surface energies of $S_{1}$ and $S_{2}$, respectively (see Figure 3). These energies may depend on each point of the boundary of $D$. Afterwards, on the domain $S_{1}^{\prime}$, the surface energy between gas and solid is neglected [Adamson 1967]. The liquid drop is submitted to a volume force $\rho \boldsymbol{f}$. The external surface force on $D$ is modelized with two constraint vector fields, $\boldsymbol{T}_{1}$ on the solid surface, $S_{1}$, and $\boldsymbol{T}_{2}$ on the free surface, $S_{2}$. The line tension $\boldsymbol{L}$ is assumed to be null.

By using the principle of virtual work, we aim to write the equation of motion of the liquid drop and the conditions on the surfaces and line bordering the liquid drop.

3.2. Surfactant attached to interfacial fluid particles. To express the behavior of the surface energy, we need to represent first the equation of the surface matter density.

Under the mapping $\varphi$, the set $D_{0}$ with boundary $S_{0}$ has image $D$ with boundary $S$. We assume there exists an insoluble surfactant with a surface mass concentration $c_{0}$ defined on $D_{0}$ of image $c$ in $D$ [Levitch 1962; Adamson 1967; Defay 1971]. 
Let us consider the case when the surfactant is attached to the fluid particles on the surface $S$, that is,

$$
c_{0}=c_{0}(\boldsymbol{X})
$$

where $\boldsymbol{X} \in S_{0}$.

The mass conservation of the surfactant on the surface $S$ requires that for any subset $S_{0}^{*}$ of $S_{0}$, of image $S^{*}$ subset of $S$,

$$
\iint_{S^{*}} c d s=\iint_{S_{0}^{*}} c_{0} d s_{0}
$$

Relation (9) implies

$$
c \operatorname{det} F \boldsymbol{n}_{0}^{T} F^{-1} \boldsymbol{n}=c_{0} \quad \text { with } \boldsymbol{n}_{0}^{T}=\frac{\boldsymbol{n}^{T} F}{\sqrt{\left(\boldsymbol{n}^{T} F F^{T} \boldsymbol{n}\right)}},
$$

where $\boldsymbol{n}_{0}$ denotes the unit normal vector to $S_{0}$. The proof of (10) is given in the Appendix.

From (10), we obtain the following: Firstly, the conservation of the surface concentration of the surfactant,

$$
\frac{d c}{d t}+c\left(\operatorname{div} \boldsymbol{u}-\boldsymbol{n}^{T} \boldsymbol{D} \boldsymbol{n}\right)=0,
$$

where $\boldsymbol{u}$ is the fluid velocity vector and

$$
\boldsymbol{D}=\frac{1}{2}\left(\partial \boldsymbol{u} / \partial \boldsymbol{x}+(\partial \boldsymbol{u} / \partial \boldsymbol{x})^{T}\right)
$$

denotes the rate of the deformation tensor of the fluid. The term $\operatorname{div} \boldsymbol{u}-\boldsymbol{n}^{T} \boldsymbol{D} \boldsymbol{n}$ expresses the tangential divergence relative to the surface $S$.

Secondly, the variation of the mass concentration of the surfactant,

$$
\delta c+c\left[\operatorname{div} \zeta-\boldsymbol{n}^{T} \frac{\partial \boldsymbol{\zeta}}{\partial \boldsymbol{x}} \boldsymbol{n}\right]=0 .
$$

The proofs of (11) and (12) are also given in the Appendix. In the case when the surface energy $\sigma$ is a function of the surfactant concentration,

$$
\sigma=\sigma(c),
$$

we deduce $\delta \sigma=\sigma^{\prime}(c) \delta c$. If we denote

$$
\gamma=\sigma-c \sigma^{\prime}(c)
$$

which is the Legendre transformation of $\sigma$ with respect to $c$, then by taking (A.3) into account we obtain, in the Appendix,

$$
\delta E=-\iint_{S}\left[\frac{2 \gamma}{R_{m}} \boldsymbol{n}^{T}+\operatorname{grad}^{T} \gamma\left(\mathbf{1}-\boldsymbol{n} \boldsymbol{n}^{T}\right)\right] \zeta d s+\int_{\Gamma} \gamma \boldsymbol{n}^{\prime T} \zeta d l .
$$


As we shall see in Section $4, \gamma$ is the surface tension of the interface $S$. The variation $\delta c$ of the concentration has an important consequence on the surfactant behavior and the surfactant behavior is essential to determining the virtual work of the liquid drop.

Relation (13) can easily be extended to several surfactants: if

$$
\sigma=\sigma\left(c_{1}, \ldots, c_{n}\right),
$$

where the $c_{i}$, for $i \in\{1, \ldots, n\}$, are the concentrations of the $n$ surfactants, then

$$
\gamma=\sigma-\sum_{i=1}^{n} c_{i} \frac{\partial \sigma}{\partial c_{i}},
$$

corresponding to the Legendre transformation of $\sigma$ with respect to $c_{i}, i \in\{1, \ldots, n\}$ and (14) is always valid.

3.3. Governing equation of motion and boundary conditions. As previously indicated, we do not consider the thermodynamical problem of interfaces, but, for example, when the medium is isothermal, $\alpha$ can be considered as the specific free energy of the bulk and $\sigma$ the free surface energy of the interface.

The use of virtual displacements yields a linear functional of virtual works, the sum of several partial works. To enumerate the works of forces, we have to consider how they are obtained in the literature [Germain 1973a; 1973b; Gouin 2007]. The virtual work expressions of volume force $\rho \boldsymbol{f}$, surface force $\boldsymbol{T}$, and liquid pressure $p$ are the same as in Section 2.2.

(a) For fluid motion, the virtual work of mass impulsions is

$$
-\iiint_{D} \rho \boldsymbol{a}^{T} \zeta d v
$$

where $\boldsymbol{a}$ is the acceleration vector.

(b) For dissipative motion, we must add the virtual work of viscous stresses

$$
-\iiint_{D} \operatorname{tr}\left(\boldsymbol{\tau}_{v} \frac{\partial \zeta}{\partial \boldsymbol{x}}\right) d v
$$

where $\boldsymbol{\tau}_{v}$ denotes the viscous stress tensor usually written in Navier-Stokes form [Adamson 1967]. Taking account of the relation

$$
\operatorname{tr}\left(\boldsymbol{\tau}_{v} \frac{\partial \zeta}{\partial \boldsymbol{x}}\right)=\operatorname{div}\left(\boldsymbol{\tau}_{v} \zeta\right)-\left(\operatorname{div} \boldsymbol{\tau}_{v}\right) \zeta
$$

an integration by parts using Stokes' formula in (14) for the virtual work of interfacial forces, together with the relations $\boldsymbol{n}_{1}^{\prime}=\boldsymbol{n}_{1} \times \boldsymbol{t}$ and $\boldsymbol{n}_{2}^{\prime}=-\boldsymbol{n}_{2} \times \boldsymbol{t}$, allows us 
to obtain the virtual work of forces applied to the domain $D$ :

$$
\begin{aligned}
\delta \tau & =\iiint_{D}\left(-\operatorname{grad}^{T} p+\operatorname{div} \boldsymbol{\tau}_{v}+\rho \boldsymbol{f}^{T}-\rho \boldsymbol{a}^{T}\right) \zeta d v \\
& +\iint_{S_{1}}\left[\operatorname{grad}^{T} \gamma_{1}\left(\mathbf{1}-\boldsymbol{n}_{1} \boldsymbol{n}_{1}^{T}\right)+\left(p+\frac{2 \gamma_{1}}{R_{m_{1}}}\right) \boldsymbol{n}_{1}^{T}-\boldsymbol{n}_{1}^{T} \boldsymbol{\tau}_{v}+\boldsymbol{T}_{1}^{T}\right] \zeta d s \\
& +\iint_{S_{2}}\left[\operatorname{grad}^{T} \gamma_{2}\left(\mathbf{1}-\boldsymbol{n}_{2} \boldsymbol{n}_{2}^{T}\right)+\left(p+\frac{2 \gamma_{2}}{R_{m_{2}}}\right) \boldsymbol{n}_{2}^{T}-\boldsymbol{n}_{2}^{T} \boldsymbol{\tau}_{v}+\boldsymbol{T}_{2}^{T}\right] \zeta d s \\
& +\int_{\Gamma}\left(\gamma_{1} \boldsymbol{n}_{1}^{\prime T}-\gamma_{2} \boldsymbol{n}_{2}^{\prime T}\right) \zeta d l,
\end{aligned}
$$

where $R_{m_{i}}$ denotes the mean radius of curvature of $S_{i}, \gamma_{i}$ denotes the surface tension of $S_{i}$, and $\boldsymbol{T}_{i}$ the surface force on $S_{i}, i \in\{1,2\} ; \boldsymbol{T}_{2}=-P \boldsymbol{n}_{2}$, where $P$ is the pressure in the external gas to the domain $D$.

The field of virtual displacement $x \in D \longrightarrow \zeta(x) \in T_{x}(D)$ must be tangent to the solid (rigid) surface $S_{1}$. The fundamental lemma of variational calculus yields the equation of motion associated with domain $D$, the conditions on surfaces $S_{1}$ and $S_{2}$, and the condition on contact line $\Gamma$.

Due to the fact that (15) is expressed in separate form in the sense of distributions [Schwartz 1966], the d'Alembert-Lagrange principle implies that $\forall \boldsymbol{\zeta}(\boldsymbol{x}) \in T_{x}(D)$ tangent to $S_{1}$, each of the four integrals of (15) is null. Then, we obtain equations on $D, S_{1}, S_{2}$, and $\Gamma$, respectively.

- We get the equation of motion in $D$ :

$$
\rho \boldsymbol{a}+\operatorname{grad} p=\left(\operatorname{div} \boldsymbol{\tau}_{v}\right)^{T}+\rho \boldsymbol{f} .
$$

Equation (16) is the Navier-Stokes equation for compressible fluids when $\boldsymbol{\tau}_{v}$ is written in the classical linear form by using the rate of the fluid deformation tensor, $\boldsymbol{\tau}_{v}=\lambda(\operatorname{tr} \boldsymbol{D}) \mathbf{1}+2 \mu \boldsymbol{D}$. We may add a classical condition for the velocity on the boundary as the adherence condition.

- We get the condition on surface $S_{1}$. The virtual displacement is tangent to $S_{1}$; the constraint $\boldsymbol{n}_{1}^{T} \zeta=0$ implies there exists a scalar Lagrange multiplier $\boldsymbol{x} \in S_{1} \longrightarrow$ $\chi(\boldsymbol{x}) \in \Re$, such that

$$
\left(p+\frac{2 \gamma_{1}}{R_{m_{1}}}\right) \boldsymbol{n}_{1}-\boldsymbol{\tau}_{v} \boldsymbol{n}_{1}+\left(\mathbf{1}-\boldsymbol{n}_{1} \boldsymbol{n}_{1}^{T}\right) \operatorname{grad} \gamma_{1}+\boldsymbol{T}_{1}=\chi \boldsymbol{n}_{1},
$$

The normal and tangential components of (17) relative to $S_{1}$ are deduced from (17):

$$
\begin{array}{r}
p+\frac{2 \gamma_{1}}{R_{m_{1}}}-\boldsymbol{n}_{1}^{T} \boldsymbol{\tau}_{v} \boldsymbol{n}_{1}+\boldsymbol{n}_{1}^{T} \boldsymbol{T}_{1}=\chi, \\
\left(\boldsymbol{1}-\boldsymbol{n}_{1} \boldsymbol{n}_{1}^{T}\right)\left(-\boldsymbol{\tau}_{v} \boldsymbol{n}_{1}+\operatorname{grad} \gamma_{1}+\boldsymbol{T}_{1}\right)=0 .
\end{array}
$$


Following (18), we obtain the value of $\chi$ along the surface $S_{1}$. The scalar field $\chi$ corresponds to the unknown value of the normal stress vector on the surface $S_{1}$; it corresponds to the difference between the mechanical and viscous normal stresses and a stress due to the curvature of $S_{1}$ taking into account the surface tension. Equation (19) represents the balance between the tangential components of the mechanical and viscous stresses and the tangential component of the surface tension gradient.

- We get the condition on surface $S_{2}$ :

$$
\left(p+\frac{2 \gamma_{2}}{R_{m_{2}}}\right) \boldsymbol{n}_{2}-\boldsymbol{\tau}_{v} \boldsymbol{n}_{2}+\left(\mathbf{1}-\boldsymbol{n}_{2} \boldsymbol{n}_{2}^{T}\right) \operatorname{grad} \gamma_{2}-P \boldsymbol{n}_{2}=0 .
$$

The normal and tangential components of (20) relative to $S_{2}$ are deduced:

$$
\begin{aligned}
\frac{2 \gamma_{2}}{R_{m_{2}}}-\boldsymbol{n}_{2}^{T} \boldsymbol{\tau}_{v} \boldsymbol{n}_{2}+p & =P, \\
\left(\mathbf{1}-\boldsymbol{n}_{2} \boldsymbol{n}_{2}^{T}\right)\left(-\boldsymbol{\tau}_{v} \boldsymbol{n}_{2}+\operatorname{grad} \gamma_{2}\right) & =0 .
\end{aligned}
$$

Equation (21) corresponds to the expression of the Laplace equation in the case of viscous motion; the normal component of viscous stresses is taken into account. Equation (22) is similar to (19) for the surface $S_{2}$ but without the component of the stress vector.

- We get the condition on line $\Gamma$. To get the line condition we must consider a virtual displacement tangent to $S_{1}$ and consequently in the form

$$
\zeta=\alpha \boldsymbol{t}+\beta \boldsymbol{t} \times \boldsymbol{n}_{1},
$$

where $\alpha$ and $\beta$ are two scalar fields defined on $\Gamma$. From the last integral of (15), we get the following immediately: For any scalar field $\boldsymbol{x} \in \Gamma \longrightarrow \beta(\boldsymbol{x}) \in \Re$,

$$
\int_{\Gamma} \beta \gamma_{1} \boldsymbol{n}_{1}^{\prime T}\left(\boldsymbol{t} \times \boldsymbol{n}_{1}\right) d l-\int_{\Gamma} \beta \gamma_{2} \boldsymbol{n}_{2}^{\prime T}\left(\boldsymbol{t} \times \boldsymbol{n}_{1}\right) d l=0,
$$

with $\boldsymbol{n}_{1}^{\prime}=-\boldsymbol{t} \times \boldsymbol{n}_{1}$ and $\boldsymbol{n}_{2}^{\prime}=\boldsymbol{t} \times \boldsymbol{n}_{2}$, and consequently,

$$
-\gamma_{1}-\gamma_{2} \boldsymbol{n}_{2}^{T} \boldsymbol{n}_{1}=0
$$

Denoting by $\theta$ the angle $\left\langle\boldsymbol{n}_{1}, \boldsymbol{n}_{2}\right\rangle$, we obtain the well-known relation of YoungDupré but adapted to $\gamma_{1}$ and $\gamma_{2}$ in place of $\sigma_{1}$ and $\sigma_{2}$ :

$$
\gamma_{1}+\gamma_{2} \cos \theta=0 \text {. }
$$

3.4. Remarks. For a motionless fluid, $\boldsymbol{\tau}_{v}=0$ and consequently, (19) yields

$$
\operatorname{grad}_{t g} \gamma_{1}=-\boldsymbol{T}_{1 t g},
$$


where $\operatorname{grad}_{t g} \gamma_{1}$ and $\boldsymbol{T}_{1 t g}$ denote the tangential parts of $\operatorname{grad} \gamma_{1}$ and $\boldsymbol{T}_{1}$, respectively. The tangential part of the vector stress is opposite to the surface tension gradient. Therefore, at a given value of $T_{1 n}=\boldsymbol{n}_{1}^{T} \boldsymbol{T}_{1}$, (18) yields the value $\chi$ corresponding to the normal stress vector to the surface $S_{1}$, (21) yields $P=p+2 \gamma_{2} / R_{m_{2}}$ corresponding to the classical equation of Badshforth and Adams [Adamson 1967] but with the surface tension $\gamma_{2}$ instead of $\sigma_{2}$, and (22) implies $\left(\mathbf{1}-\boldsymbol{n}_{2} \boldsymbol{n}_{2}^{T}\right) \operatorname{grad} \gamma_{2}=0$. At equilibrium, along $S_{2}$, the surface tension $\gamma_{2}$ must be uniform.

In the case of motion, (22) represents the Marangoni effect as proposed in [Gibbs 1928; Defay 1971] but with the surface tension $\gamma_{2}$ instead of $\sigma_{2}$.

\section{Surface energy and surface tension}

A surface tension must appear on the boundary conditions as a force per unit of line. The Legendre transformation $\gamma$ of $\sigma$ with respect to $c$ exactly corresponds to this property on the contact line $\Gamma$; then, surface tension $\gamma$ differs from the surface energy; this important property was pointed out by Gibbs [1928] and Defay [1971] by means of thermodynamical considerations. The fundamental difference between surface tension and surface energy, in the presence of attached surfactants, is illustrated in the following cases corresponding to formal behaviors.

- If $\sigma$ is independent of $c$, then $\gamma=\sigma$ : the surface tension is equal to the surface energy. This is the classical case of capillarity for fluids considered in Section 2.2 and (23) is the classical Young-Dupré condition on the contact lines.

- In fact, $\sigma$ is a decreasing function of $c$ [Adamson 1967]; when $c$ is small enough we consider the behavior

$$
\sigma=\sigma_{0}-\sigma_{1} c, \quad \text { where } \sigma_{0}>0 \text { and } \sigma_{1}>0 ;
$$

then, (13) implies $\gamma=\sigma_{0}$ and surface tension and surface energy are different.

- Now, we consider a formal case when the surface energy density model is written in the form

$$
\sigma=\sigma_{0}-\sigma_{1} c-\sigma_{2} c \sin \left(\frac{1}{c}\right)
$$

where $\sigma_{0}>0, \sigma_{1}>0$, and $\sigma_{2}>0$. Then, (13) implies

$$
\gamma=\sigma_{0}-\sigma_{2} \cos \left(\frac{1}{c}\right) \text {. }
$$

This case does not correspond to $\sigma$ as a monotonic decreasing function of $c$. Nevertheless, when $c \rightarrow 0, \gamma$ does not have any limit and we get

$$
\gamma \in\left[\sigma_{0}-\sigma_{2}, \sigma_{0}+\sigma_{2}\right] .
$$

The surface tension may have a large scale of values. When the concentration $c$ is low, a variation of the concentration $c$ may generate strong fluctuations of 
the surface tension without significant change of the surface energy. Alternatively, the concentration behavior strongly affects the surface tension but not the surface energy. Relation (24) fits with the well-known physical case of hysteresis behavior for a drop lying on a horizontal plane (see, for example, [Gouin 2003] and the literature therein). So, the surface roughness is not the only reason of the hysteresis of the contact angle even if the surface energy is nearly constant.

\section{Conclusion}

The principle of virtual work allows us to deduce the equation of motion and the conditions on the surfaces and lines by means of a variational analysis. When capillary forces operate and surfactant molecules are attached to the fluid molecules at the interfaces, the conditions on the surfaces and lines point out a fundamental difference between the concepts of surface energy and surface tension. This fact was thermodynamically predicted in [Gibbs 1928; Defay 1971]. The hysteresis phenomenon may appear even if surface energy is almost constant on a planar substrate when the surface tension strongly varies.

In (23), $\gamma_{1}$ and $\gamma_{2}$ are not assumed to be constant, but are defined at each point of $\Gamma$. This expression of the Young-Dupré boundary condition on the contact line $\Gamma$ is not true in more complex cases. For example, in the case when the surface tension is a nonlocal functional of surfactant concentration, the surface tension is no longer the classical Legendre transformation of the surface energy relative to surfactant concentration and more complex behaviors can be foreseen. These behaviors can change the variation of the integral of the free energy as in the case of shells or in second-gradient models for which boundary conditions become more complex [Cosserat and Cosserat 1909; Toupin 1962; Germain 1973b; Noll and Virga 1990; Dell'Isola and Seppecher 1997]. In a further article [Gouin $\geq 2014$ ], we will see this is also the case when the surface energy depends on the surface curvature as in membranes and vesicles [Helfrich 1973; Seifert 1997; Agrawal and Steigmann 2011].

\section{Appendix: Geometrical preliminaries}

[Kobayashi and Nomizu 1963; Aris 1989; Gouin and Kosiński 1998]

A.1 Expression of the virtual work of forces in capillarity. The hypotheses and notations are presented in Section 2.2.

Lemma 1. We have the following relations:

$$
\begin{aligned}
\delta \operatorname{det} F & =\operatorname{det} F \operatorname{div} \zeta, \\
\delta\left(F^{-1} \boldsymbol{n}\right) & =-F^{-1} \frac{\partial \zeta}{\partial \boldsymbol{x}} \boldsymbol{n}+F^{-1} \delta \boldsymbol{n} .
\end{aligned}
$$


The proof of (A.1) comes from the so-called Jacobi identity,

$$
\delta(\operatorname{det} F)=\operatorname{det} F \operatorname{tr}\left(F^{-1} \delta F\right),
$$

and from

$$
\delta F=\delta\left(\frac{\partial \boldsymbol{x}}{\partial \boldsymbol{X}}\right)=\frac{\partial \zeta}{\partial \boldsymbol{X}}
$$

Then,

$$
\operatorname{tr}\left(F^{-1} \delta F\right)=\operatorname{tr}\left(\frac{\partial \boldsymbol{X}}{\partial \boldsymbol{x}} \frac{\partial \zeta}{\partial \boldsymbol{X}}\right)=\operatorname{tr}\left(\frac{\partial \zeta}{\partial \boldsymbol{X}} \frac{\partial \boldsymbol{X}}{\partial \boldsymbol{x}}\right)=\operatorname{tr}\left(\frac{\partial \zeta}{\partial \boldsymbol{x}}\right)=\operatorname{div} \zeta
$$

The proof of (A.2) comes as follows:

$$
\delta\left(F^{-1} \boldsymbol{n}\right)=\delta\left(F^{-1}\right) \boldsymbol{n}+F^{-1} \delta \boldsymbol{n} .
$$

But $F^{-1} F=\mathbf{1}$ implies

$$
\delta\left(F^{-1}\right) F+F^{-1} \delta F=0,
$$

and so also

$$
\delta\left(F^{-1}\right)=-F^{-1} \frac{\partial \zeta}{\partial \boldsymbol{X}} F^{-1}=-F^{-1} \frac{\partial \zeta}{\partial \boldsymbol{x}},
$$

which yields (A.2).

Lemma 2. Let us consider the surface integral

$$
E=\iint_{S} \sigma d s
$$

Then the variation of $E$ is,

$$
\delta E=\iint_{S}\left[\delta \sigma-\left(\frac{2 \sigma}{R_{m}} \boldsymbol{n}^{T}+\operatorname{grad}^{T} \sigma\left(\mathbf{1}-\boldsymbol{n} \boldsymbol{n}^{T}\right)\right) \zeta\right] d s+\int_{\Gamma} \sigma \boldsymbol{n}^{\prime T} \zeta d l .
$$

Relation (A.3) points out the extreme importance of knowing the variation of $\delta \sigma$. The variation $\delta E$ of $E$ drastically changes following the different possible behaviors of the surface energy.

The proof can be found as follows. The external normal $\boldsymbol{n}(\boldsymbol{x})$ to $S$ is locally extended in the vicinity of $S$ by the relation $\boldsymbol{n}(\boldsymbol{x})=\operatorname{grad} d(\boldsymbol{x})$, where $d$ is the distance of point $\boldsymbol{x}$ to $S$; for any vector field $\boldsymbol{w}$, we obtain [Kobayashi and Nomizu 1963; Aris 1989]

$$
\operatorname{rot}(\boldsymbol{n} \times \boldsymbol{w})=\boldsymbol{n} \operatorname{div} \boldsymbol{w}-\boldsymbol{w} \operatorname{div} \boldsymbol{n}+\frac{\partial \boldsymbol{n}}{\partial \boldsymbol{x}} \boldsymbol{w}-\frac{\partial \boldsymbol{w}}{\partial \boldsymbol{x}} \boldsymbol{n} .
$$

From $\boldsymbol{n}^{T} \frac{\partial \boldsymbol{n}}{\partial \boldsymbol{x}}=0$ and $\operatorname{div} \boldsymbol{n}=-\frac{2}{R_{m}}$, we deduce on $S$,

$$
\boldsymbol{n}^{T} \operatorname{rot}(\boldsymbol{n} \times \boldsymbol{w})=\operatorname{div} \boldsymbol{w}+\frac{2}{R_{m}} \boldsymbol{n}^{T} \boldsymbol{w}-\boldsymbol{n}^{T} \frac{\partial \boldsymbol{w}}{\partial \boldsymbol{x}} \boldsymbol{n} .
$$


Due to

$$
E=\iint_{S} \sigma \operatorname{det}\left(\boldsymbol{n}, d_{1} \boldsymbol{x}, d_{2} \boldsymbol{x}\right),
$$

where $d_{1} \boldsymbol{x}$ and $d_{2} \boldsymbol{x}$ are differential vectors associated with two coordinate lines of $S$, we get

$$
E=\iint_{S_{0}} \sigma \operatorname{det} F \operatorname{det}\left(F^{-1} \boldsymbol{n}, d_{1} \boldsymbol{X}, d_{2} \boldsymbol{X}\right),
$$

where $d_{1} \boldsymbol{x}=F d_{1} \boldsymbol{X}$ and $d_{2} \boldsymbol{x}=F d_{2} \boldsymbol{X}$. Then,

$\delta E=\iint_{S_{0}} \delta \sigma \operatorname{det} F \operatorname{det}\left(F^{-1} \boldsymbol{n}, d_{1} \boldsymbol{X}, d_{2} \boldsymbol{X}\right)+\iint_{S_{0}} \sigma \delta\left(\operatorname{det} F \operatorname{det}\left(F^{-1} \boldsymbol{n}, d_{1} \boldsymbol{X}, d_{2} \boldsymbol{X}\right)\right)$.

Due to Lemma 1 and the fact that $\boldsymbol{n}^{T} \boldsymbol{n}=1$ implies $\boldsymbol{n}^{T} \delta \boldsymbol{n}=0$,

$$
\begin{aligned}
& \iint_{S_{0}} \sigma \delta\left(\operatorname{det} F \operatorname{det}\left(F^{-1} \boldsymbol{n}, d_{1} \boldsymbol{X}, d_{2} \boldsymbol{X}\right)\right) \\
& =\iint_{S}\left[\sigma \operatorname{div} \zeta \operatorname{det}\left(\boldsymbol{n}, d_{1} \boldsymbol{x}, d_{2} \boldsymbol{x}\right)+\sigma \operatorname{det}\left(\delta \boldsymbol{n}, d_{1} \boldsymbol{x}, d_{2} \boldsymbol{x}\right)-\sigma \operatorname{det}\left(\frac{\partial \zeta}{\partial \boldsymbol{x}} \boldsymbol{n}, d_{1} \boldsymbol{x}, d_{2} \boldsymbol{x}\right)\right] \\
& =\iint_{S}\left(\operatorname{div}(\sigma \zeta)-\left(\operatorname{grad}^{T} \sigma\right) \zeta-\sigma \boldsymbol{n}^{T} \frac{\partial \zeta}{\partial \boldsymbol{x}} \boldsymbol{n}\right) d s .
\end{aligned}
$$

Relation (A.4) yields

$$
\operatorname{div}(\sigma \zeta)+\frac{2 \sigma}{R_{m}} \boldsymbol{n}^{T} \zeta-\boldsymbol{n}^{T} \frac{\partial \sigma \zeta}{\partial \boldsymbol{x}} \boldsymbol{n}=\boldsymbol{n}^{T} \operatorname{rot}(\sigma \boldsymbol{n} \times \zeta)
$$

Then,

$$
\begin{aligned}
\iint_{S_{0}} \sigma \delta\left(\operatorname{det} F \operatorname{det}\left(F^{-1} \boldsymbol{n}, d_{1} \boldsymbol{X}, d_{2} \boldsymbol{X}\right)\right) \\
=\iint_{S}\left(-\frac{2 \sigma}{R_{m}} \boldsymbol{n}^{T}+\operatorname{grad}^{T} \sigma\left(\boldsymbol{n} \boldsymbol{n}^{T}-\mathbf{1}\right)\right) \zeta d s+\iint_{S} \boldsymbol{n}^{T} \operatorname{rot}(\sigma \boldsymbol{n} \times \zeta) d s,
\end{aligned}
$$

where $\operatorname{grad}^{T} \sigma\left(\boldsymbol{n} \boldsymbol{n}^{T}-\mathbf{1}\right)$ belongs to the cotangent plane to $S$ and we obtain (A.3).

A.1.1 Variation of the internal energy. Let us note that

$$
\delta \iiint_{D} \rho \alpha d v=\iiint_{D} \rho \delta \alpha d v, \quad \text { where } \delta \alpha=\frac{\partial \alpha}{\partial \rho} \delta \rho .
$$

Due to the mass conservation,

$$
\rho \operatorname{det} F=\rho_{0}(\boldsymbol{X}),
$$

where $\rho_{0}$ is defined on $D_{0}$, the differentiation of (A.5) yields

$$
\delta \rho \operatorname{det} F+\rho \delta(\operatorname{det} F)=0,
$$


and from Lemma 1 we get

$$
\delta \rho=-\rho \operatorname{div} \zeta .
$$

Consequently, from $p=\rho^{2} \frac{\partial \alpha}{\partial \rho}$ and $\operatorname{div}(p \zeta)=p \operatorname{div} \zeta+(\operatorname{grad} p)^{T} \zeta$, we get

$$
\begin{aligned}
\delta \iiint_{D} \rho \alpha d v & =\iiint_{D} \rho \frac{\partial \alpha}{\partial \rho} \delta \rho d v=\iiint_{D}-p \operatorname{div} \zeta d v \\
& =\iiint_{D}(\operatorname{grad} p)^{T} \zeta d v-\iint_{S} p \boldsymbol{n}^{T} \zeta d s .
\end{aligned}
$$

By taking into account (A.3), we immediately get (4).

\section{A.2 Study of a surfactant attached to fluid particles.}

A.2.1 Proof of (10). Under the hypotheses and notations of Section 3.2,

$$
\begin{aligned}
\iint_{S^{*}} c d s & =\iint_{S^{*}} \operatorname{det}\left(\boldsymbol{n} c, d_{1} \boldsymbol{x}, d_{2} \boldsymbol{x}\right)=\iint_{S_{0}^{*}} \operatorname{det}\left(F F^{-1} \boldsymbol{n} c, F d_{1} \boldsymbol{X}, F d_{2} \boldsymbol{X}\right) \\
& =\iint_{S_{0}^{*}} c(\operatorname{det} F) \operatorname{det}\left(F^{-1} \boldsymbol{n}, d_{1} \boldsymbol{X}, d_{2} \boldsymbol{X}\right)=\iint_{S_{0}^{*}} c(\operatorname{det} F) \boldsymbol{n}_{0}^{T} F^{-1} \boldsymbol{n} d s_{0},
\end{aligned}
$$

where $\boldsymbol{n}_{0}^{T} \boldsymbol{n}_{0}=1$. Moreover, $\boldsymbol{n}^{T} d \boldsymbol{x}=0 \Rightarrow \boldsymbol{n}^{T} F d \boldsymbol{X}=0$, then $\boldsymbol{n}_{0}^{\prime T}=\boldsymbol{n}^{T} F$ is normal to $S_{0}^{*}$, and consequently,

$$
\boldsymbol{n}_{0}^{T}=\frac{\boldsymbol{n}^{T} F}{\sqrt{\left(\boldsymbol{n}^{T} F F^{T} \boldsymbol{n}\right)}}, \quad \boldsymbol{n}^{T}=\frac{\boldsymbol{n}_{0}^{T} F^{-1}}{\sqrt{\left(\boldsymbol{n}_{0}^{T} F^{-1}\left(F^{-1}\right)^{T} \boldsymbol{n}_{\mathbf{0}}\right)}},
$$

and from (9),

$$
c \operatorname{det} F \sqrt{\boldsymbol{n}_{0}^{T} F^{-1}\left(F^{-1}\right)^{T} \boldsymbol{n}_{0}}=c_{0} .
$$

A.2.2 Proof of (11) and (12). With the notations of Section 3.2, (A.7) yields

$$
\frac{d c}{d t}=-\frac{c_{0} \frac{d(\operatorname{det} F)}{d t}}{(\operatorname{det} F)^{2} \sqrt{\left(\boldsymbol{n}_{0}^{T} F^{-1}\left(F^{-1}\right)^{T} \boldsymbol{n}_{0}\right)}}-\frac{c_{0} \frac{d}{d t}\left(\boldsymbol{n}_{0}^{T} F^{-1}\left(F^{-1}\right)^{T} \boldsymbol{n}_{0}\right)}{2 \operatorname{det} F\left(\boldsymbol{n}_{0}^{T} F^{-1}\left(F^{-1}\right)^{T} \boldsymbol{n}_{0}\right)^{3 / 2}} .
$$

But, $\frac{d(\operatorname{det} F)}{d t}=(\operatorname{det} F) \operatorname{div} \boldsymbol{u}$ and $\frac{d}{d t}\left(F^{-1}\left(F^{-1}\right)^{T}\right)=-2 F^{-1} \boldsymbol{D}\left(F^{-1}\right)^{T}$. Then,

$$
\frac{d c}{d t}+c\left(\operatorname{div} \boldsymbol{u}-\boldsymbol{n}^{T} \boldsymbol{D} \boldsymbol{n}\right)=0 .
$$

The same calculation with $\delta$ in place of $\frac{d}{d t}$ yields immediately

$$
\delta c+c\left[\operatorname{div} \zeta-\boldsymbol{n}^{T} \frac{\partial \zeta}{\partial \boldsymbol{x}} \boldsymbol{n}\right]=0
$$


A.2.3 Proof of (14). From (12) and $\sigma=\sigma(c)$ we get

$$
\delta \sigma=\kappa\left[\operatorname{div} \zeta-\boldsymbol{n}^{T} \frac{\partial \zeta}{\partial \boldsymbol{x}} \boldsymbol{n}\right], \quad \text { with } \kappa(c)=-c \sigma^{\prime}(c) .
$$

Consequently,

$$
\iint_{S} \delta \sigma=\iint_{S}\left(\operatorname{div}(\kappa \zeta)-\operatorname{grad}^{T} \kappa \zeta-\kappa \boldsymbol{n}^{T} \frac{\partial \zeta}{\partial \boldsymbol{x}} \boldsymbol{n}\right) d s
$$

But (A.4) implies

$$
\boldsymbol{n}^{T} \operatorname{rot}(\kappa \boldsymbol{n} \times \zeta)=\operatorname{div}(\kappa \zeta)+\frac{2 \kappa}{R_{m}} \boldsymbol{n}^{T} \zeta-\boldsymbol{n}^{T} \frac{\partial(\kappa \zeta)}{\partial \boldsymbol{x}} \boldsymbol{n},
$$

and

$$
\boldsymbol{n}^{T} \frac{\partial(\kappa \zeta)}{\partial \boldsymbol{x}} \boldsymbol{n}=\left(\boldsymbol{n}^{T} \zeta\right) \cdot\left(\operatorname{grad}^{T} \kappa \boldsymbol{n}\right)+\kappa \boldsymbol{n}^{T} \frac{\partial \zeta}{\partial \boldsymbol{x}} \boldsymbol{n}=\operatorname{grad}^{T} \kappa \boldsymbol{n} \boldsymbol{n}^{T} \zeta+\kappa \boldsymbol{n}^{T} \frac{\partial \zeta}{\partial \boldsymbol{x}} \boldsymbol{n} .
$$

Then,

$\operatorname{div}(\kappa \zeta)-\operatorname{grad}^{T} \kappa \zeta-\kappa \boldsymbol{n}^{T} \frac{\partial \zeta}{\partial \boldsymbol{x}} \boldsymbol{n}=-\frac{2 \kappa}{R_{m}} \boldsymbol{n}^{T} \zeta-\operatorname{grad}^{T} \kappa\left(\mathbf{1}-\boldsymbol{n} \boldsymbol{n}^{T}\right) \zeta+\boldsymbol{n}^{T} \operatorname{rot}(\kappa \boldsymbol{n} \times \zeta)$.

Due to

$$
\iint_{S} \boldsymbol{n}^{T} \operatorname{rot}(\kappa \boldsymbol{n} \times \zeta) d s=\int_{\Gamma} \kappa \boldsymbol{n}^{T} \zeta d l
$$

we get

$$
\iint_{S}-\delta \sigma d s=\iint_{S}\left[\frac{2 \kappa}{R_{m}} \boldsymbol{n}^{T}+\operatorname{grad}^{T} \kappa\left(\mathbf{1}-\boldsymbol{n} \boldsymbol{n}^{T}\right)\right] \zeta d s-\int_{\Gamma} \kappa \boldsymbol{n}^{\prime T} \zeta d l,
$$

and (A.3) yields

$$
\delta E=-\iint_{S}\left[\frac{2 \gamma}{R_{m}} \boldsymbol{n}^{T}+\operatorname{grad}^{T} \gamma\left(\mathbf{1}-\boldsymbol{n} \boldsymbol{n}^{T}\right)\right] \zeta d s+\int_{\Gamma} \gamma \boldsymbol{n}^{\prime T} \zeta d l .
$$

\section{Acknowledgements}

I am indebted to the three anonymous referees for much valuable criticism, comments, and suggestions during the review process.

\section{References}

[Adamson 1967] A. W. Adamson, Physical chemistry of surfaces, Interscience, New York, 1967.

[Agrawal and Steigmann 2011] A. Agrawal and D. J. Steigmann, "A model for surface diffusion of trans-membrane proteins on lipid bilayers", Z. Angew. Math. Phys. 62:3 (2011), 549-563.

[Aris 1989] R. Aris, Vectors, tensors, and the basic equations of fluid mechanics, Dover, New York, 1989. 
[Cermelli et al. 2005] P. Cermelli, E. Fried, and M. E. Gurtin, "Transport relations for surface integrals arising in the formulation of balance laws for evolving fluid interfaces", J. Fluid Mech. 544 (2005), 339-351.

[Cosserat and Cosserat 1909] E. Cosserat and F. Cosserat, Sur la théorie des corps déformables, Hermann, Paris, 1909.

[Daher and Maugin 1986] N. Daher and G. A. Maugin, "The method of virtual power in continuum mechanics. Application to media presenting singular surfaces and interfaces", Acta Mech. 60:3-4 (1986), 217-240.

[Defay 1971] R. Defay, Thermodynamique de la tension superficielle, Gauthier-Villars, Paris, 1971.

[Dell'Isola and Seppecher 1997] F. dell'Isola and P. Seppecher, "Edge contact forces and quasibalanced power", Meccanica 32:1 (1997), 33-52.

[Domb 1996] C. Domb, The critical point, Taylor \& Francis, London, 1996.

[Edwards et al. 1991] D. A. Edwards, H. Brenner, and D. T. Wasan, Interfacial transport processes and rheology, Butterworth-Heinemann, Stoneham, 1991.

[Fischer et al. 2008] F. D. Fischer, T. Waitz, D. Vollath, and N. K. Simha, "On the role of surface energy and surface stress in phase-transforming nanoparticles", Progress in Materials Science 53:3 (2008), 481-527.

[Fosdick and Tang 2009] R. Fosdick and H. Tang, "Surface transport in continuum mechanics", Mathematics and Mechanics of Solids 14:6 (2009), 587-598.

[de Gennes et al. 2004] P. G. de Gennes, F. Brochard-Wyart, and D. Quéré, Capillarity and wetting phenomena: drops, bubbles, pearls, waves, Springer, Berlin, 2004.

[Germain 1973a] P. Germain, "La méthode des puissances virtuelles en mécanique des milieux continus. I. Théorie du second gradient”, J. Mécanique 12 (1973), 235-274.

[Germain 1973b] P. Germain, "The method of the virtual power in continuum mechanics, 2: Microstructure", SIAM J. Appl. Math. 25 (1973), 556-575.

[Gibbs 1928] J. W. Gibbs, The scientific papers of J. Willard Gibbs, vol. 1, Longmans, London, 1928.

[Gouin 2003] H. Gouin, "The wetting problem of fluids on solid surfaces, II: The contact angle hysteresis”, Contin. Mech. Thermodyn. 15:6 (2003), 597-611.

[Gouin 2007] H. Gouin, "The d'Alembert-Lagrange principle for gradient theories and boundary conditions", pp. 79-95 in Asymptotic methods in nonlinear wave phenomena, World Sci. Publ., Hackensack, NJ, 2007.

[Gouin $\geq 2014$ ] H. Gouin, "Vesicles: a capillarity model revisited", in preparation.

[Gouin and Kosiński 1998] H. Gouin and W. Kosiński, "Boundary conditions for a capillary fluid in contact with a wall”, Arch. Mech. (Arch. Mech. Stos.) 50:5 (1998), 907-916.

[Helfrich 1973] W. Helfrich, "Elastic properties of lipid bilayers: theory and possible experiments", Z. Naturforsch. C 28 (1973), 693-703.

[Kobayashi and Nomizu 1963] S. Kobayashi and K. Nomizu, Foundations of differential geometry, vol. 1, Interscience, London, 1963.

[Levitch 1962] V. Levitch, Physicochemical hydrodynamics, Prentice-Hall, New Jersey, 1962.

[McBride et al. 2011] A. T. McBride, A. Javili, P. Steinmann, and S. Bargmann, "Geometrically nonlinear continuum thermomechanics with surface energies coupled to diffusion", J. Mech. Phys. Solids 59:10 (2011), 2116-2133.

[Noll and Virga 1990] W. Noll and E. G. Virga, "On edge interactions and surface tension", Arch. Rational Mech. Anal. 111:1 (1990), 1-31. 
[Ono and Kondo 1960] S. Ono and S. Kondo, "Molecular theory of surface tension in liquids", pp. 134-280 in Handbuch der Physik, Bd. X, Springer, Berlin, 1960.

[Rocard 1952] Y. Rocard, Thermodynamique, Masson, Paris, 1952.

[Rosen 2004] M. J. Rosen, Surfactants and interfacial phenomena, Wiley, New Jersey, 2004.

[Schwartz 1966] L. Schwartz, Théorie des distributions, Publications de l'Institut de Mathématique de l'Université de Strasbourg, No. IX-X. Nouvelle édition, entiérement corrigée, refondue et augmentée, Hermann, Paris, 1966.

[Seifert 1997] U. Seifert, "Configurations of fluid membranes and vesicles", Adv. Phys. 46 (1997), $13-137$.

[Serrin 1959] J. Serrin, "Mathematical principles of classical fluid mechanics", pp. 125-263 in Handbuch der Physik (herausgegeben von S. Flügge), Bd. 8/1, Strömungsmechanik I (Mitherausgeber C. Truesdell), Springer, Berlin, 1959.

[Slattery et al. 2007] J. C. Slattery, L. Sagis, and E.-S. Oh, Interfacial transport phenomena, Second ed., Springer, New York, 2007.

[Steigmann and Li 1995] D. J. Steigmann and D. Li, "Energy-minimizing states of capillary systems with bulk, surface, and line phases", IMA J. Appl. Math. 55:1 (1995), 1-17.

[Steinmann 2008] P. Steinmann, “On boundary potential energies in deformational and configurational mechanics”, J. Mech. Phys. Solids 56:3 (2008), 772-800.

[Toupin 1962] R. A. Toupin, "Elastic materials with couple-stresses", Arch. Rational Mech. Anal. 11 (1962), 385-414.

Received 5 Nov 2012. Revised 15 Mar 2013. Accepted 14 Apr 2013.

HENRI GOUIN: henri.gouin@univ-amu.fr

Aix-Marseille Université, CNRS, Centrale Marseille, M2P2 UMR 7340, 13451, Marseille, France 


\section{EDITORIAL BOARD}

ANTONIO CARCATERRA ERIC A. CARLEN

FRANCESCO DELL'ISOLA

RAFFAELE ESPOSITO

ALBERT FANNJIANG

Gilles A. FrancFort

PiERANGElo MARCATI

JEAN-JACQUES MARIGO

PETER A. MARKOWICH MARTIN OSTOJA-STARZEWSKI

PIERRE SEPPECHER

DAVID J. STEIGMANN

PAUl STEINMANN

PierRe M. Suquet

\section{MANAGING EDITORS}

MICOL AMAR

CORRADO LATTANZIO

ANGELA MADEO

MARTIN OSTOJA-STARZEWSKI

\section{ADVISORY BOARD}

ADNAN AKAY

Holm AltenbaCH

MICOL AMAR

HARM ASKES

TEODOR ATANACKOVIĆ

VICTOR BERDICHEVSKY

GuY BouchitTÉ

ANDREA BRAIDES

ROBERTO CAMASSA

MAURO CARFORE

ERIC DARVE

FELIX DARVE

ANNA DE MASI

Gianpietro Del Piero

EMMANUELE Di BENEDETTO

BERNOLD FIEDLER

IRENE M. GAMBA

SERGEY GAVRILYUK

TIMOTHY J. HEALEY

DOMINIQUE JEULIN

ROGER E. KHAYAT

CORRADO LATTANZIO

ROBERT P. LIPTON

ANGELO LUONGO

ANGEla MadeO

JUAN J. MANFREDI

CARLO MARCHIORO

GÉrard A. MAUGin

ROBERTO NATALINI

PATRIZIO NEFF

ANDREY PIATNITSKI

ERrico Presutti

MARIO PUlVIRENTI

LuCiO RuSSO

Miguel A. F. SANJUAN

Patrick SElvadurai

ALEXANDER P. SEYRANIAN

MIROSLAV ŠILHAVÝ

GUIDO SWEERS

ANTOINETTE TORDESILLAS

LEV TRUSKINOVSKY

JUAN J. L. VELÁZQUEZ

VINCENZO VESPRI

ANGELO VULPIANI msp.org/memocs

Università di Roma “La Sapienza”, Italia

Rutgers University, USA

(CO-CHAIR) Università di Roma "La Sapienza", Italia

(TREASURER) Università dell'Aquila, Italia

University of California at Davis, USA

(CO-CHAIR) Université Paris-Nord, France

Università dell' Aquila, Italy

École Polytechnique, France

DAMTP Cambridge, UK, and University of Vienna, Austria

(CHAIR MANAGING EDITOR) Univ. of Illinois at Urbana-Champaign, USA

Université du Sud Toulon-Var, France

University of California at Berkeley, USA

Universität Erlangen-Nürnberg, Germany

LMA CNRS Marseille, France

Università di Roma “La Sapienza”, Italia

Università dell' Aquila, Italy

Université de Lyon-INSA (Institut National des Sciences Appliquées), France (CHAIR MANAGING EDITOR) Univ. of Illinois at Urbana-Champaign, USA

Carnegie Mellon University, USA, and Bilkent University, Turkey

Otto-von-Guericke-Universität Magdeburg, Germany

Università di Roma "La Sapienza”, Italia

University of Sheffield, UK

University of Novi Sad, Serbia

Wayne State University, USA

Université du Sud Toulon-Var, France

Università di Roma Tor Vergata, Italia

University of North Carolina at Chapel Hill, USA

Università di Pavia, Italia

Stanford University, USA

Institut Polytechnique de Grenoble, France

Università dell'Aquila, Italia

Università di Ferrara and International Research Center MEMOCS, Italia

Vanderbilt University, USA

Freie Universität Berlin, Germany

University of Texas at Austin, USA

Université Aix-Marseille, France

Cornell University, USA

École des Mines, France

University of Western Ontario, Canada

Università dell' Aquila, Italy

Louisiana State University, USA

Università dell' Aquila, Italia

Université de Lyon-INSA (Institut National des Sciences Appliquées), France

University of Pittsburgh, USA

Università di Roma "La Sapienza", Italia

Université Paris VI, France

Istituto per le Applicazioni del Calcolo "M. Picone", Italy

Universität Duisburg-Essen, Germany

Narvik University College, Norway, Russia

Università di Roma Tor Vergata, Italy

Università di Roma "La Sapienza", Italia

Università di Roma “Tor Vergata", Italia

Universidad Rey Juan Carlos, Madrid, Spain

McGill University, Canada

Moscow State Lomonosov University, Russia

Academy of Sciences of the Czech Republic

Universität zu Köln, Germany

University of Melbourne, Australia

École Polytechnique, France

Bonn University, Germany

Università di Firenze, Italia

Università di Roma La Sapienza, Italia

MEMOCS (ISSN 2325-3444 electronic, 2326-7186 printed) is a journal of the International Research Center for the Mathematics and Mechanics of Complex Systems at the Università dell'Aquila, Italy.

Cover image: "Tangle" by @ John Horigan; produced using the Context Free program (contextfreeart.org).

\section{PUBLISHED BY}

mathematical sciences publishers

nonprofit scientific publishing

http://msp.org/

(C) 2014 Mathematical Sciences Publishers 
Mathematics and Mechanics of Complex Systems vol. 2 no. 1

Delaminated thin elastic inclusions inside elastic bodies

Alexander M. Khludnev and Günter R. Leugering

Interfaces endowed with nonconstant surface energies

23 revisited with the d'Alembert-Lagrange principle

Henri Gouin

Particles for fluids: SPH versus vortex methods

Andrea Colagrossi, Giorgio Graziani and Mario

Pulvirenti

Nonclassical continua, pseudobalance, and the law of action 71 and reaction

Gianpietro Del Piero

MEMOCS is a journal of the International Research Center for the Mathematics and Mechanics of Complex Systems at the Università dell' Aquila, Italy.

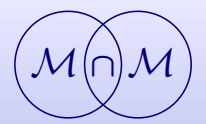

